\section{Treatment Escalation Plan Quality Improvement Project}

Poster presented at BAPIO National Conference, London Nov 2019 Peer reviewed by Subarna Chakravorty \& Sunil Daga
Nikhil Aggarwal

Cite as: Aggarwal, N. Treatment Escalation Plan Quality Improvement Project. The Physician 2020 vol 6; issue 2: abp16 DOI: 10.38192/1.6.2.23

Article Information

Epub: 27.11.2019

Published21.08.2020

Open Access- Creative Commons Licence CCBY-ND-4.0

Keywords:

Treatment escalation

Background:

Treatment escalation describes the level of treatment intervention which would be appropriate, should the patient become acutely unwell. Patients may be subjected to inappropriate investigations and management by the 'on call' team as well as inappropriate referrals to ITU/HDU because there is often no clear documentation regarding the 'ceiling of care.' Furthermore, documentation of a treatment escalation plan (TEP) is often difficult to find and vague.

Methods:

Data was collected from three care of the elderly (COE) wards for the presence of a documented TEP in the notes. A TEP proforma was designed through collaboration with staff. The agreed version was to be filled out by doctors, grade registrar and above.

It was trialled on the same three CoE wards for four weeks. Post-intervention data was collected on rates and location of TEP documentation. Qualitative feedback was sought from staff.

\section{Results:}

Pre-intervention, data was collected from 58 patient notes, 22 patients (37.9\%) had a documented TEP. The location of the TEP varied. The documentation for $100 \%$ of patients simply stated "ward based ceiling of care."

Following our intervention, data was collected from 48 patient notes and 35 patients $(60.4 \%)$ had a documented TEP. The proforma was used in $100 \%$ of cases with a documented TEP.

Overall, staff felt that the proforma was quick to fill out, clear and useful.
The main issue raised was having to complete an additional form as well as the DNAR form.

Conclusion:

The new TEP proforma has improved documentation rates of TEPs providing clarity when dealing with deteriorating patients and therefore improving patient care.

The next step will be to modify and review the proforma with consultation from the legal department to merge the DNAR and TEP proformas.

This proforma is a major step in maintaining and improving patient safety.

Conflict of Interests/Comments: Nothing to declare. 